\title{
THE APPLICATIONS OF STEREOLITHOGRAPHY IN FACIAL RECONSTRUCTIVE SURGERY
}

\author{
CHEUNG L.K. ${ }^{1}$, WONG M.C.M. ${ }^{2}$, WONG L.L.S. ${ }^{3}$ \\ Oral \& Maxillofacial Surgery, University of Hong Kong ${ }^{1}$ \\ Industrial Centre, The Hong Kong Polytechnic University ${ }^{2}$ \\ Department of Radiology, Queen Mary Hospital ${ }^{3}$
}

\begin{abstract}
The development of rapid prototyping has evolved from the crude milled models to the laser polymerised stereolithographic models of excellent accuracy. The technology was advanced further with the recent introduction of fused deposition. modelling and the 3-dimensional ink-jet printing technique in stereo-model fabrication. The concept of using a 3dimensional model in planning the operation has amazed the maxillofacial surgeons since its first application in grafting a skull defect in 1995. It was followed by many bright ideas of applications in the field of facial reconstructive surgery. Stereo-model may assist in diagnosis of facial fractures, joint ankylosis and even impacted teeth. The surgery can be simulated prior the operation of complex craniofacial syndromes, facial asymmetry and distraction osteogenesis. The stereomodel can be used for preparation of reconstructive plate or joint prosthesis. It has an enormous value as an educational teaching and patient information tool for obtaining the consent for surgery.
\end{abstract}

Keywords:

computer model; reconstructive surgery; maxillofacial surgery

\section{Introduction}

Three-dimensional models of diseases and deformities can be traced back to an artistic tradition in medicine and surgery. The most famous examples are the "moulages" made of coloured wax representing skin diseases, deformities and tumour. However, their reconstruction was extremely time-consuming and only a few experts were initiated into the secret of this profession. Until recently the only 3dimensional representation of craniofacial deformities could be found in skull collections, gathered for demonstration of specific anatomical anomalies. The introduction of reproducible and precise models of selected bone structures in medicine is very recent. The possibility of generating 3-dimensional models from CT scans was first mentioned in $1980^{1}$. The first craniofacial model was produced in foam by milling method ${ }^{4}$. The most spectacular technique in the innovationorientated field of imaging is stereolithography ${ }^{11}$. Modeling has become so reliable with precision reaching $98 \%{ }^{3}$ that maxillofacial surgeons can use the stereomodels for planning of complicated facial reconstructive surgery. However, the manufacturing method of stereolithographic models is expensive and time consuming, which limits its availability to most surgeons.

The aims of the paper are to present the modern manufacturing methods of stereomodel and to illustrate the clinical applications of stereomodel in facial reconstruction.

\section{Stereolithography}

Stereolithography (SLA) was the first rapid prototyping technology available in the market. The SLA machine builds the 
stereo-models from liquid photosensitive polymers that solidify when exposed to ultraviolet light beam. The model is built upon a platform situated just below the surface in a vat of liquid epoxy or acrylic resin. A low power highly focused ultraviolet laser traces out the first layer, sodifying the model's cross section while leaving the remaining area in liquid form. Upon completion of each cross sectional polymerization, the model platform lowers to the next incremental level into the liquid polymer. A sweeper re-coats the solidified layer with liquid, and the laser traces the second layer on top of the first layer. This process is repeated until the prototype is complete.

On completion of model forming process, the unpolymerized liquid polymer is drained leaving the solid model that can be delivered out of the vat. The model is then cleansed under running water. The supporting resin pillars created during the model forming process are removed. The model is placed inside an ultraviolet oven for the final curing.

\section{Fused Deposition Modelling}

In this method of fused deposition modelling (FDM), a thread of thermoplastic filament in semi-solid state is extruded out from a heated nozzle that moves on a horizontal platform. The platform is kept at a lower temperature than the nozzle, so that the filament deposited on the platform will be hardened quickly. The nozzle is controlled to weave the pattern required by the computer. After each horizontal layer is formed, the platform will be lowered in the vertical direction. The nozzle will deposit the next layer upon the previous one. A model will be built in layers after layers according to the computer data. In order to prevent dropping of the overhanging structure, another kind of thermoplastic material is used to form supporting pillars, which can be easily removed from the main model.

\section{Three-dimensional printing}

An entire different class of machines that employ ink-jet technology fabricates the 3dimensional printing (3DP) model. The models are built on a platform, which contains a bin full of powder material. An ink-jet printing head ejects the binding liquid selectively according to the computer data in order to fuse the powder together to form a solid model. The unbound powder surrounding the model will support the integrity of the model, without the need to have supporting pillars. The platform is lowered while more power is added over the next crosssectional layer to be bound. The process is repeated until a complete model is formed. The model is then carefully removed from the bin of powder. The surface of the model is painted with a coat of cyanoacrylate liquid for improvement of its surface tensile strength.

\section{Comparison of manufacturing methods}

The SLA and FDM require about two days to form a skull model in contrast to about 6 hours by 3DP method. The machine cost in forming the SLA model is also about 3.5 times more than the FDM and 3DP. This makes the SLA model most expensive, followed by FDM and then the 3DP model. On the other hand, the tensile and flexural strength of the SLA model is considerably higher than the other two types of model. The model formed by 3DP is most fragile, particularly when it is made from starch. The post-processing with cyanoacrylate paint will increase the tensile strength of the starch model from 435 psi to 2900 psi. However, the strength is still considerable below that of the SLA of 11,100 psi and the FDM of 5,000 psi. 
The main advantage of the 3DP is that the model can be quickly formed within a few. hours to cope with the clinical requirement such as emergency craniofacial fracture treatment.

\section{Clinical applications in facial reconstruction}

\section{Diagnosis}

The stereomodel provides a 3dimensional information of the complex facial skeleton. This is particularly useful in differentiating bony overlaps near the skull base region, such as jaw joint (condylar) fracture, where traditional radiography cannot provide quality image. In mid-facial fractures, extensive comminution of the facial skeleton can be extremely confusing on the cross-sectional CT images. A 3-D reformatted image or the availability of a stereo-model will provide the surgeons enormous insight on the complex fracture pattern ${ }^{7}$. In addition, 3-D model is also useful in determining the extent of bony ankylosis of the jaw joint and to highlight any important adjacent vital structures by the use of coloured SLA. In cleidocranial dysplasia where the patient has multiple supernumerary teeth, the use of selective coloured stereomodel will even provide the 3-D localization of the abnormal teeth $^{10}$.

\section{Surgical simulation}

This is the aspect of stereomodelling that attracts the maxillofacial surgeons most, where multiple applications have been reported. Simulation surgery can be performed on the stereomodel to check its feasibility and to predict the surgical outcome. It is more indicated for surgical planning of craniofacial syndromes rather than orthognathic surgery, where the metallic orthodontic brackets may even confuse the dental landmarks ${ }^{5,6,8}$. Traditional plaster models mounted on articulator are more suitable for routine orthognathic surgery.

The planned vector of movement is the key component of success in distraction osteogenesis (Fig. 1). It is extremely useful to have the stereomodel available for the planning of the distractor location and angulation in order to ensure a reasonable prediction of the final dental occlusion and facial symmetry?

\section{Reconstruction of defects}

Surgical repair of skull defect can be planned on the stereomodel by fabrication of custom-made template to cover the defect or the use of image copying technique in reconstructing the defect with the opposite normal skull bone ${ }^{2}$. Bone graft may also be adapted on the sterilized stereomodel during the operation and before its transplantation to the facial skeleton.

In tumour surgery where part of the facial bones is to be resected, pre-bending of the reconstruction plate on the stereomodel is useful in reducing the operating hours. In patients who have lost the condyle from previous surgery, the temporomandibular joint prosthesis can also be adapted before the operation (Fig. 2). The availability of the stereomodel can reduce both the surgical and anaesthetic time, which will ultimately benefit the patients with less surgical morbidity.

\section{Patient information}

The stereomodel can provide patients with extensive 3-dimensional information on the anatomy or pathology of the facial skeleton. The surgical approach in carrying out the operation is much easier to be understood by the patients, which is of growing importance in the signing of consent for operation.

In a teaching hospital, stereomodels form excellent educational tool for the teaching of students in the appreciation of the 
anatomy, pathology, surgical access and technique in the reconstructive surgery.

\section{Conclusion}

Stereomodels can be manufactured by different modern methods with an attempt to reduce both the cost and time, while maintaining the anatomical reproduction accuracy and usefulness. The applications of stereomodel in facial reconstructive surgery increase considerably in recent years, which include diagnosis, surgical simulation, reconstructive planning, and patient information. The main advantages are reduction of surgical time, and morbidity of the patients as well as improving the quality of the surgical results.

\section{References}

[1] Alberti C. Three-dimensional CT and structure models. Br J Radiol 1980: 53; 261-2.

[2] Bill J.S., Reuther J.F., Dittmann W., Kübler N., Meier II J.L., Pistner G. Stereolithography in oral and maxillofacial operation planning. Int J Oral Maxillofac Surg 1995: 24; 98-103.

[3] Bouyssié J.F., Bouyssié S., Sharrock P., Durab D. Stereolithographic models derived from x-ray computed tomography reproduction accuracy. Surg Radiol Anat 1997: 19; 193-9.

[4] Brix F., Lambrecht J.T.

Individuelle Schädelmodellherstellung auf der Grundlage computertomographischer Informationen. Fortschr. Kiefer-Gesichts Chir 1987: 32; 74-7.

[5] Hibi H., Sawaki Y., Ueda M.

Three-dimensional model simulation in orthognathic surgery. Int $\mathbf{J}$ Adult Orthod Orthognath Surg 1997: 12; 226-32.
[6] James W.J., Slabbekoorn M.A., Edgin W.A., Hardin C.K.

Correction of congenital malar hypoplasia using stereolithography for presurgical planning. J Oral Maxillofac Surg 1998: 56; 512-7.

[7] Kermer C., Rasse M., Lagogianis G., Undt G., Wagner A., Millesi W. Colour stereolithography for planning complex maxillofacial tumour surgery. J CranioMax Fac Surg 1998: 26; 360-2.

[8] Sailer H.F., Haers P.E., Zollikofer C.P.E., Warnke T., Carls F.R., Stucki P. The value of stereolithographic models for preoperative diagnosis of craniofacial deformities and planning of surgical corrections. Int J Oral Maxillofac Surg 1998: 27; 327-33.

[9] Santler G., Kärcher H., Ruda C. Indications and limitations of threedimensional models in cranio-maxillofcial surgery. J Cranio-Max Fac Surg 1998: 26; 11-6.

[10] Sato K., Sugawara J., Mitani H., Kawamura $\mathrm{H}$. Use of selectively colored stereolithography for diagnosis of impacted supernumerary teeth for a patient with cleidocranial dysplasia. Int $\mathbf{J}$ Adult Orthod Orthognath Surg 1998: 13; 163-7.

[11] Zonneveld F.W., Noorman van der Dussen M.F. Three-dimensional imaging and model fabrication in oral and maxillofacial surgery. Oral maxillofac Surg Clin N Am 1992: 4; 19-33.

Corresponence Address:

Professor Lim K. Cheung

Oral \& Maxillofacial Surgery

The University of Hong Kong

Prince Philip Dental Hospital

34 Hospital Road, Hong Kong 
Figure 1. Surgical simulation surgery in lengthening of mandibular ramus in a girl with hemifacial microsomia.
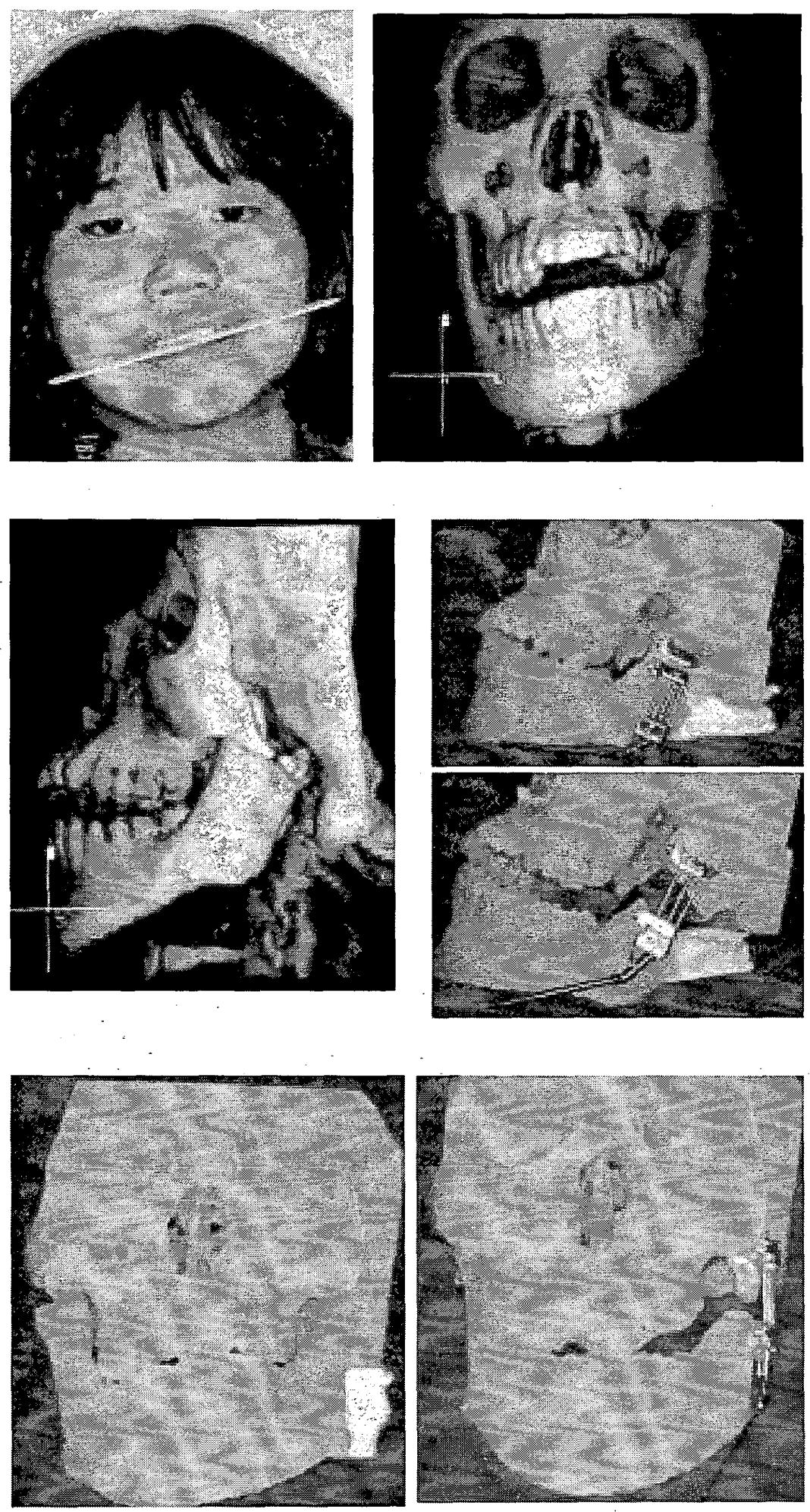
Figure 2. Reconstruction of temporomandibular joints in a patient who lost the condyles from previous surgery.
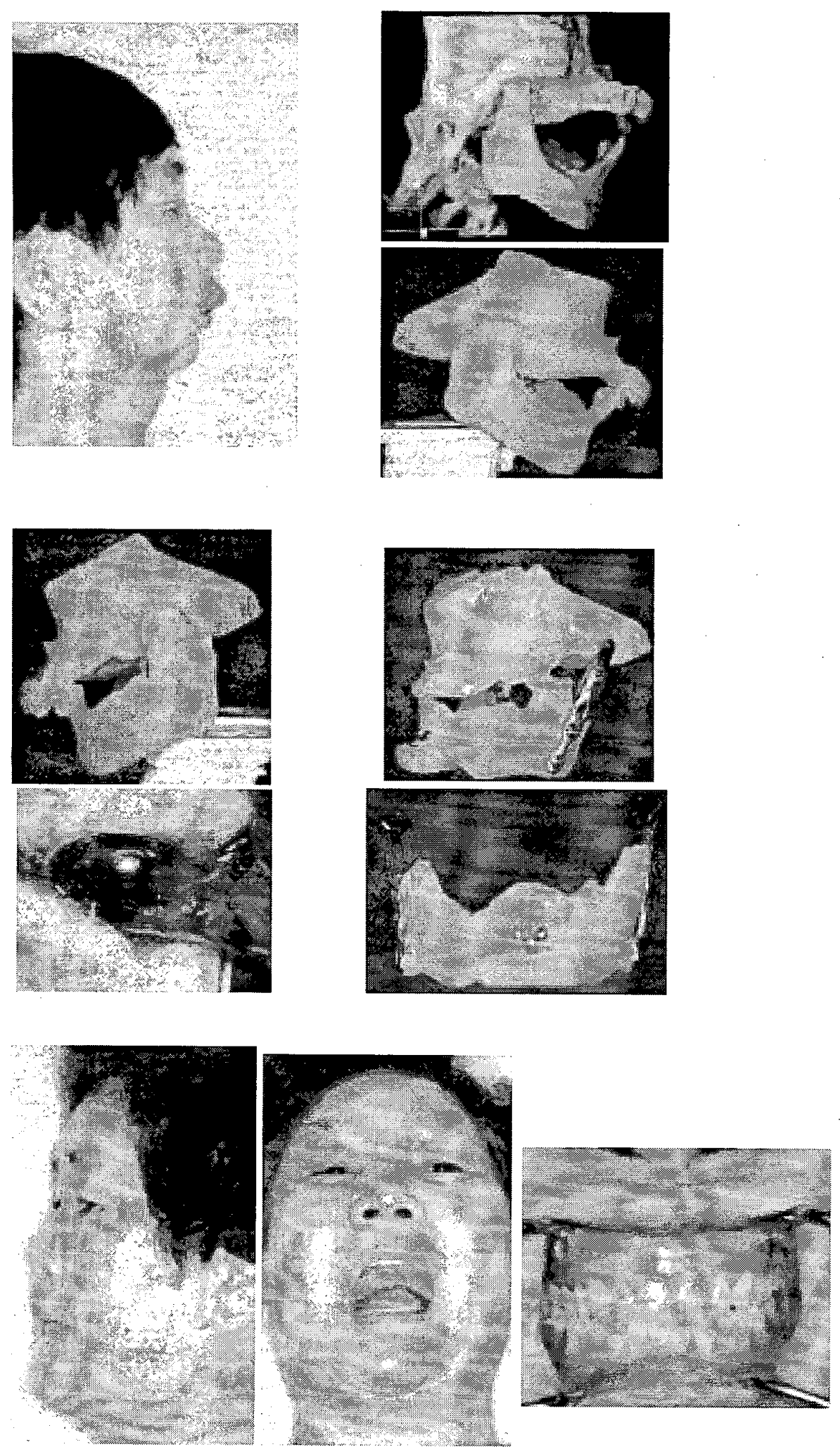